\title{
Effect of the Double-Line Spiral Tunnel Curvature on the Tunnel Construction Stability
}

\author{
Chao Zhang, ${ }^{1}$ Chao Wang, ${ }^{1}$ Xibin Niu, ${ }^{1}$ Shaoqiang Zhang, ${ }^{1}$ and Huiling Zhao $\mathbb{D}^{2}$ \\ ${ }^{1}$ PowerChina Roadbridge Group Co., Ltd., Beijing 100048, China \\ ${ }^{2}$ Department of Civil Engineering, Shanghai University, Shanghai 200444, China \\ Correspondence should be addressed to Huiling Zhao; hlzhao@shu.edu.cn
}

Received 29 July 2020; Revised 19 November 2020; Accepted 30 November 2020; Published 17 December 2020

Academic Editor: Wen-Chieh Cheng

Copyright (c) 2020 Chao Zhang et al. This is an open access article distributed under the Creative Commons Attribution License, which permits unrestricted use, distribution, and reproduction in any medium, provided the original work is properly cited.

In the construction of mountain highway, in order to avoid complicated geology and adapt to the requirements of the terrain of the large height difference and the large slope, the double-line spiral tunnels are gradually applied. The purpose of this paper is to analyze the mechanical behavior of the double-line spiral tunnel and its surrounding rock under different line curvatures, and to obtain the influence of the curvature of the spiral tunnel on the stability of the double-line tunnel construction. The analysis of this paper is based on the engineering background of double-line spiral tunnels in China's Yunnan province. The elastoplastic threedimensional rock strata-tunnel by means of finite difference FLAC3D software was established to simulate the construction process. The model was verified by comparing the calculation results and the actual monitoring data of tunnel vault settlement. The small curvature radius spiral makes the mechanical behavior of the double-line tunnel uneven and the surrounding rock deformed unevenly. A quantitative analysis and qualitative evaluation of the influence of curvature radius were established by the systematic evaluation index of (1) ratio of compressive stress on both sides of the tunnel, (2) stress ratio of double-line tunnel, (3) convergent deformation of the cross section of the tunnel, and (4) deformation of the surrounding rock on the top of the tunnel. The results show that the small curvature radius (less than $200 \mathrm{~m}$ ) will make the inner pressure of the inner tunnel significantly greater than the external pressure stress, showing obvious asymmetry, and the inner tunnel vault tensile stress is greater than the outer tunnel. With the increase of the curvature radius (about more than $400 \mathrm{~m}$ ), the ratio of the compressive stress on the inside and outside of the tunnel tends to be constant, and the bias condition is weakened and stabilized. Meanwhile, the smaller curvature radius makes the convergent deformation of the cross section of the tunnel appear asymmetrical, and the compression quantity inside the tunnel center line is larger. It provides a reference basis for the stability control of the construction of the double-line spiral tunnels in the mountainous area.

\section{Introduction}

The mountainous area of the plateau tends to be characterized by the deep valley and high mountain. In order to avoid poor geology and adapt to the requirements of large height difference and large longitudinal slope terrain, the tunnel alignment is usually designed to be spiral [1-3]. The alignment of the spiral tunnel is composed of continuous codirectional curves. Due to the limitation of the terrain, obstacles, and the engineering investment, the curvature of the tunnel axis line is required to be tight [4]. Besides, the tunnel is designed as a two-way double-line to improve the traffic capacity effectively [5]. The construction technology of double-line spiral curve tunnels with the small curvature radius in rock has gradually attracted attention. The deformation and stability of the surrounding rock during the excavation are critical to the safety of tunnel construction. It is significant to clarify the influence of the curvature of the double-line tunnel on the surrounding rock deformation under the specified geological conditions, and to predict the deformation range and deformation amount of the excavation face and near area.

Hao [6] found that the asymmetry of ground-surface settlement profile caused by the tunnel excavation is related to the curved alignment of the tunnel by observations. Under the dual action of asymmetric overcutting and 
construction loadings, the surface settlement of the curved tunnel is larger than that of the straight-line tunnel [7]. Huynh studied an innovated mechanized tunneling technology to construct a twin spiral tunnel at once by multicircular face shield, and also the cross section can be changed from a horizontal double circular shape to a vertical one [8]. Numerical simulations have been explored to study the stratum disturbance caused by the construction of a tunnel at curved alignment [9-12]. Mitsutaka et al. employed the proposed three-dimensional FEM kinematic model to compute immediate ground movements by a curved shield tunnel excavation in multilayered ground [13]. Li et al. [14] focused on the stress distribution of segments for a curved shield tunnel during construction, and segment stress variation from curvature of tunnel alignment, and deviation of the Jack. However, most of the above studies aimed at the curved shield tunnel in soft soil. Little literature was found to study the curved or spiral tunnels in rock. In the mountainous area of west China, tunnels are mostly constructed in rock. Ganhaizi Tunnel on Yalu Highway is a typical spiral twin tunnel in rock, and the curvature radius of the left and right lines is $600 \mathrm{~m}$ and $618 \mathrm{~m}$, respectively [15]. Such type of tunnels in rock is widely constructed recently [16]. Systematic numerical simulations of a series of tunnels with different curvatures at curved alignments need to explore asymmetric mechanical behaviors of the single spiral. Also, the differences between the twin spiral tunnels at double lines need to be paid attention. There is a slight difference in curvature.

In this paper, based on the tunnel project in Honghe, Yunnan, a 3D elastoplastic numerical model for double-line spiral tunnels in rock was established. The model was verified by the on-site monitoring data. The deformation of the surrounding rock caused by the excavation of the doubleline tunnels with the curvature radius from $122 \mathrm{~m}$ to $722 \mathrm{~m}$ was calculated. The stress and deformation distribution of the tunnels were studied and compared to obtain the asymmetric behaviors of the double-line spiral tunnels. The influence of the curvature radius on tunnel arch settlement, lateral convergence deformation, and tunnel stress distribution was analyzed. This result provides reference and guidance for safety control of tunnel design and construction in similar engineering cases.

\section{Analysis of Double-Line Spiral Tunnel Excavation Process}

2.1. Characteristics of Spiral Tunnel. The double-line spiral tunnel studied in this paper is in China's Yunnan province. The left-line tunnel has a total length of $3900 \mathrm{~m}$ and a curvature radius of $703 \mathrm{~m}$. The right-line tunnel has a total length of $4015 \mathrm{~m}$ and a curvature radius of $741 \mathrm{~m}$. The longitudinal gradient of the unidirectional slope of the tunnel is $3.9 \%$. The tunnel alignment at the entrance is the direction of $13^{\circ}$, and the tunnel alignment at the exit is the direction of $167^{\circ}$. The tunnel is deeply buried, and the maximum buried depth is $366 \mathrm{~m}$. The height difference between the tunnel entrance and exit is about $80 \mathrm{~m}$. The left line tunnel was excavated firstly, then the right line was excavated. The excavation faces of the two tunnels are about $80 \mathrm{~m}$ apart during the construction process. The rock through which the tunnel passes is mainly granite.

2.2. Establishment of 3D Numerical Model. The interval of $\mathrm{K} 36+395 \sim \mathrm{K} 36+545$ in the double-line spiral tunnel is selected as the object of three-dimensional numerical simulation analysis. The length of the tunnel alignment is $150 \mathrm{~m}$. The outer diameter of the single tunnel is $12.08 \mathrm{~m}$, and the clear distance between the left and right tunnels is $25 \mathrm{~m}$. The depth of the tunnel center is $215 \mathrm{~m}$. The surrounding rock of the tunnel is of grade III. The rock range considered in the numerical model is $3-5$ times the outer diameter of the single tunnel. The width and height dimensions of the threedimensional rock-tunnel model are $130 \mathrm{~m}$ and $110 \mathrm{~m}$, respectively. The $X$-axis is the transverse direction of the initial section of the tunnel, the $Y$-direction is the tunnel axis direction, and the $Z$-axis is the vertical direction. The bottom boundary of the numerical model is fixed, and the lateral boundary constraint is normally horizontal translation. The numerical calculation model is shown in Figure 1. The thickness of the primary lining of the tunnel is $10 \mathrm{~cm}$, and the thickness of the secondary lining is $35 \mathrm{~cm}$. During the construction of the tunnel, $\varphi 44 \mathrm{~mm}$ bolts were used for support, with a bolt depth of $2.5 \mathrm{~m}$ and a spacing of $1.45 \mathrm{~m}$.

The Mohr-Coulomb constitutive model was adopted for the rock mass, while the elastic material model was adopted for the concrete tunnel lining and the anchor rod. The material parameters are shown in Table 1.

In order to eliminate the influence of complicate initial stress distribution, the initial stress of the rock in the numerical model was simplified. The initial stress of the original rock was a geostatic field type, on which the set of vertical initial stress was based. Both horizontal stresses were proportional to the vertical stress, and the proportionality factor is 0.5 . The ground water was not considered in the simulation. The rock mass was a uniform rock stratum without consideration of tectonic activities in the rock. The parallel integrated contact was set between the different rock layers. In the simulation, the tunnel excavation was a stepby-step excavation. The single excavation step completed $3 \mathrm{~m}$ excavation forward. The excavated rock and soil were set as empty model. The lining and anchor support structure were established during the excavation.

2.3. Comparison with the Actual Monitoring Data. The tunnel crown settlements were monitored when the right line tunnel was excavated to the points YK21+640 m, $\mathrm{YK} 21+680 \mathrm{~m}, \quad \mathrm{YK} 21+700 \mathrm{~m}$, and YK21+720 $\mathrm{m}$ during construction. The monitoring results were compared with the results obtained by numerical simulation in order to verify the numerical simulation in this paper. As shown in Figure 2, the vertical ordinate indicates the amount of settlement of the vault, and the abscissa ordinate indicates the number of construction days from the excavation face. It can be seen from Figure 2 that the settlement of the tunnel vault from the $1^{\text {st }}$ to $8^{\text {th }}$ day developed faster, then it got slowly in 8-15 days, and the final cumulative settlement was 


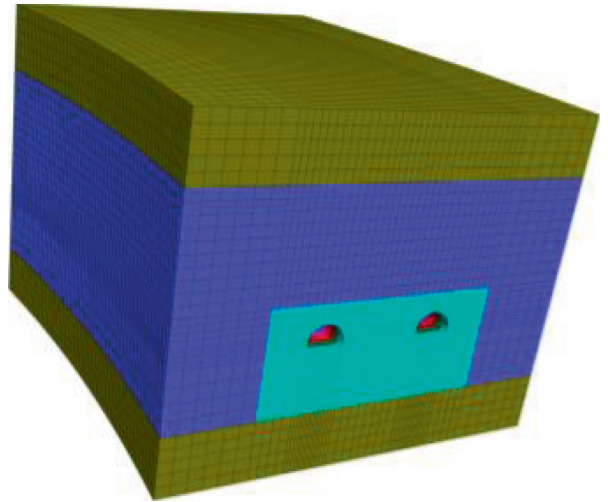

FIGURE 1: A numerical model for tunnel excavation simulation in FLAC3D.

TABLe 1: The material parameters of the tunnel numerical model.

Rock

Cohesion $(\mathrm{kPa})$

Friction angle $\left(^{\circ}\right)$ Volumetric weight $\left(\mathrm{kNm}^{-3}\right)$ Elastic modulus (GPa) Poisson's ratio 900 45 26.5 6.5 0.20

Anchor

Stiffness of cement slurry per unit length $(\mathrm{MPa})$ 17.6

Elastic modulus $(\mathrm{GPa})$ 210

Elastic modulus (GPa)

10
Initial lining

Initial lining
0.2 Elastic modulus (GPa) 10
Cross-sectional area $\left(\mathrm{mm}^{2}\right)$ 380

\section{Secondary lining}

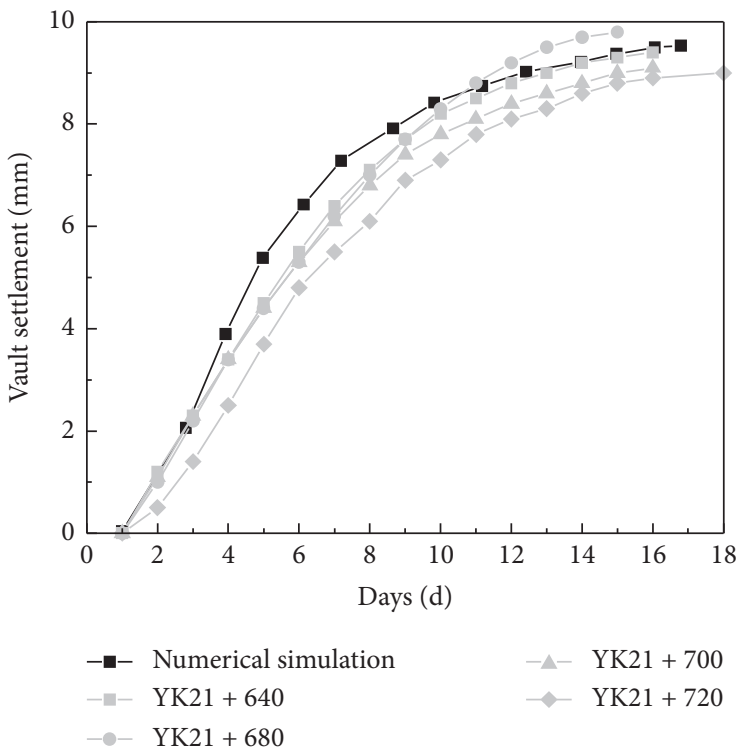

Figure 2: Comparison of numerical simulation and monitoring of tunnel vault settlement.

in the range of $8-14 \mathrm{~mm}$. The development of settlement by numerical simulation is similar to the monitoring settlement, and the overall trend is relatively consistent. The average deviation between the maximum settlement simulation result and the monitoring data is about $11 \%$. Except for accidental construction factors, the numerical simulation result is reasonable and credible.
2.4. Comparison with the Analytical Solution. The analytical methods offer a rapid analysis of the stress and strain fields and are practical for predicting the mechanical behaviors of the tunnel and the surrounding rock. The calculation model of the shallow tunnels is more complicated than the deep tunnels, which are so distant from the ground surface that the stress boundary condition is equal to the initial stress 
$[17,18]$. As for the shallow double-tunnel, the elastic analytical solution can be obtained by the complex variable method together with the Schwartz alternating method $[19,20]$. In the references $[21,22]$, the elastic region in the $z$-plane was mapped conformally onto a circular ring in the $\zeta$-plane, bounded by the circles $|\zeta|=1$ and $|\zeta|=\alpha$, as shown in Figure 3. The conformal mapping transformation is

$$
z=\omega(\zeta)=-i h \frac{1-\partial^{2}}{1+\partial^{2}} \frac{1+\zeta}{1-\zeta} .
$$

By the $\zeta$-plane, the complex variable solution for a circular tunnel in an elastic half plane was obtained under boundary conditions of the stress-free surface and the assumed displacement around the tunnel. And then the effect of the interaction between the two parallel tunnels was calculated by the Schwarz alternating method on one tunnel to another iteratively to satisfy the boundary conditions. The solution is limited to the tunnels with the circular cross section. Few analytical methods were conducted for the shallow double-tunnel with three-centered arch cross section. What is more, this paper focuses on the effect of the alignment of the spiral tunnel on the tunnel and surrounding rock. Three-dimensional elastic body with the double uncircular holes brings about challenge to the analytic solution. Therefore, this paper turns to the numerical analysis. Conventionally, the stability of tunnel structures can be analyzed with, e.g., finite element method and discrete element method [23-26]. In the paper, Fu's [21] analytic solution of shallow double tunnels in two-dimensional plane is used for a preliminary estimation and validation for the numerical analysis in some degree. Figure 4 shows the surface settlement curves by the analysis results by the $3 \mathrm{D}$ numerical model in Section 2.2 and Fu's 2D analytic solution for shallow circular double-tunnel, respectively. The two curves are close to each other. By the numerical analysis, due to the spiral tunnel alignment, the settlement of the left tunnel side is larger than the right tunnel side, while the $2 \mathrm{D}$ analytic solution is incapable of considering the effect of the spiral alignment.

\section{Influence of Curvature on Mechanical Behaviors of Tunnels}

3.1. Parametric Analysis of Numerical Experiments. In order to systematically analyze the influence of the curvature radius of the double-line spiral tunnel on the deformation and stress of the tunnel and surrounding rock, seven models with different curvature radii of tunnel alignment were set for numerical simulations, as shown in Table 2 . The variation range of curvature radius in the table was selected based on the actual project, and 7 equal interval levels were determined to conduct parametric analysis.

The quantitative analysis and qualitative evaluation of the influence of the curvature radius on the tunnel construction stability were comprehensively analyzed to obtain the influence of the curvature radius on the unevenness of the mechanical behavior of the double-line tunnel and the overall surrounding rock. Four indicators were selected for stability evaluation as1) compressive stress ratio of both sides at cross section of a single spiral tunnel, (2) stress ratio of the two tunnels at double-line, (3) tunnel cross section convergence deformation, and (4) rock deformation on the top of tunnels. Index 1 reflects the influence of curvature radius on the stress asymmetry between the inner and outer sides of the tunnel lining. Index 2 reflects the influence of curvature radius on the stress asymmetry between the left and right tunnel linings. Index 3 reflects the influence of curvature radius on the deformation unevenness of tunnel. Index 4 reflects the influence of curvature radius on the deformation tendency of surrounding rock.

\subsection{Influence on Stress Asymmetry of Tunnel Lining.} Figure 5 shows the minimum principal stress cloud diagram of the tunnel lining when the curvature radius $R$ of the center line of the double-line spiral tunnel is $122 \mathrm{~m}, 422 \mathrm{~m}$, and $722 \mathrm{~m}$. The asymmetry of the internal and external stresses is significant when $R$ is $122 \mathrm{~m}$. The asymmetry of the tunnel stress is not obvious when $R$ is $422 \mathrm{~m}$ and $722 \mathrm{~m}$.

Figure 6 shows the maximum principal stress cloud diagram of the tunnel lining when the curvature radius $R$ is $122 \mathrm{~m}, 422 \mathrm{~m}$, and $722 \mathrm{~m}$. When $R$ is $122 \mathrm{~m}$, the tensile stresses of the left and right tunnel arches at the beginning of the excavation are significantly different. When $R$ is $422 \mathrm{~m}$ and $722 \mathrm{~m}$, the difference in tensile stress between the left and right tunnels is not obvious.

The curve of stress ratio varying with curvature radius in Figure 7 is to quantify the influence of the curvature radius on the stress unevenness of the tunnel. As shown in Figure 7(a), when $R$ is $122 \mathrm{~m}$, the compressive stress ratio between the inside and outside of the left tunnel lining is the largest, reaching 1.38. As the curvature radius increases, the ratio of the inner and outer compressive stresses of the left line gradually decreases. When the curvature radius is greater than $422 \mathrm{~m}$, the ratio approaches 1.1. The compressive stress inside the left tunnel is always greater than the compressive stress outside. When $R$ is $122 \mathrm{~m}$, the ratio of the compressive stress between the inside and the outside of the lining of the right line tunnel is the largest, reaching 1.09. At this time, the compressive stress on the inside is greater than the outside. As the curvature radius increases, the ratio of the inner and outer compressive stresses on the right line gradually decreases. When the curvature radius increases to $322 \mathrm{~m}$, the ratio is less than 1.0. At this time, the inner compressive stress is less than the outer compressive stress, and the final ratio approaches 0.95 .

As for the double-line spiral tunnel, the tensile stress at the vault of the inner (left) line tunnel is always greater than that of the outer line tunnel. When $R$ is $122 \mathrm{~m}$, the tensile stress ratio of the inner and outer line lining is the largest, reaching 1.14. As the curvature radius increases, the ratio gradually decreases, as shown in Figure 7(b). It can be seen that there is a significant difference in the unevenness and distribution of the compressive stress on the inside and outside of the left and right tunnels, which should be paid attention to in the design and construction of the tunnel. 


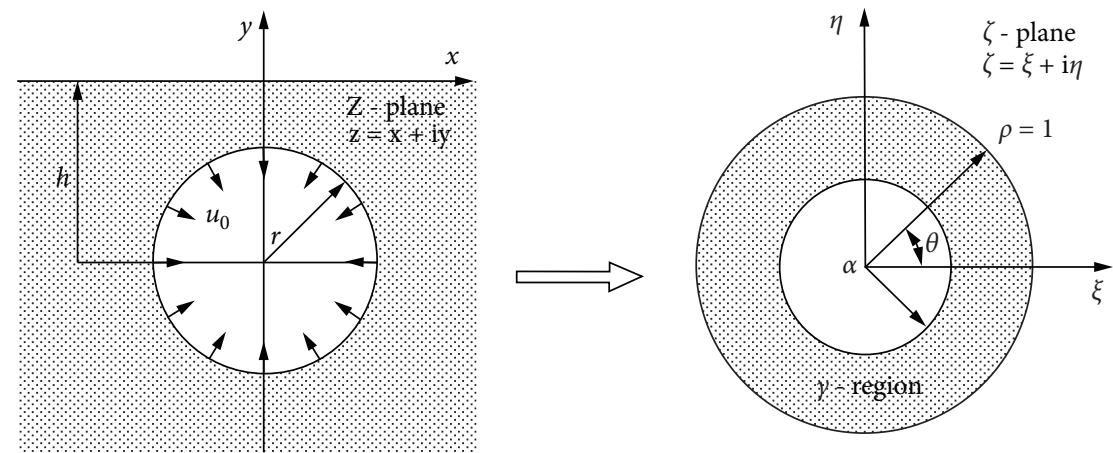

Figure 3: Conformal mapping from $z$-plane to $\zeta$-plane [17].

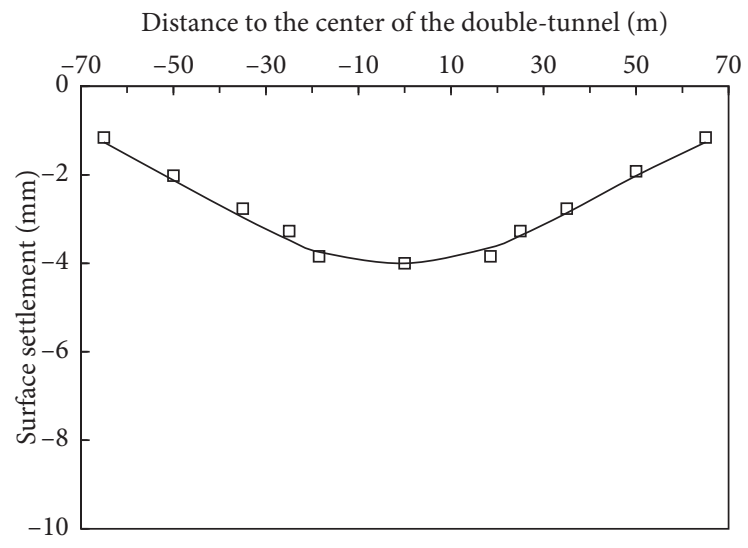

口 Analytic solution

- Numerical analysis

FIGURE 4: Comparison between the numerical results and Fu's analytic solution.

TABLE 2: Different curvature radius of the tunnel alignment for numerical simulations.

\begin{tabular}{lccc}
\hline Model no. & Curvature radius of left line $-R_{\mathrm{L}} / \mathrm{m}$ & Curvature radius of right line $R_{\mathrm{R}} / \mathrm{m}$ & Center line radius of two line- $R / \mathrm{m}$ \\
\hline 1 & 103 & 141 & 122 \\
2 & 203 & 241 & 222 \\
3 & 303 & 341 & 322 \\
4 & 403 & 441 & 422 \\
5 & 503 & 541 & 522 \\
6 & 603 & 641 & 622 \\
7 & 703 & 741 & 722 \\
\hline
\end{tabular}

Shell minimum principal stress

Cut plane: on back

Surface $-\mathrm{X}=(1,0,0)$
Depth factor $=0$

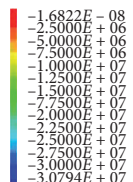

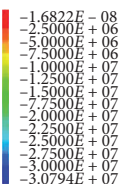

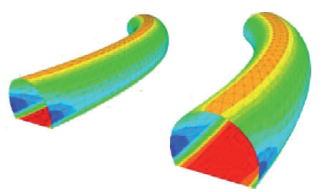

$\mathrm{R}=122 \mathrm{~m}$

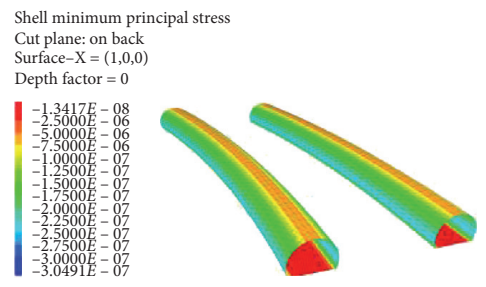

$\mathrm{R}=422 \mathrm{~m}$

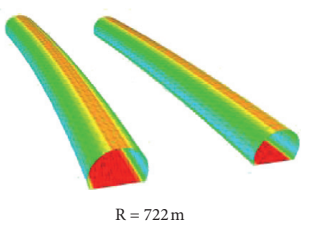

$\mathrm{R}=722 \mathrm{~m}$

FIGURE 5: Minimum principal stress of tunnels with different curvature radii (Pa). 


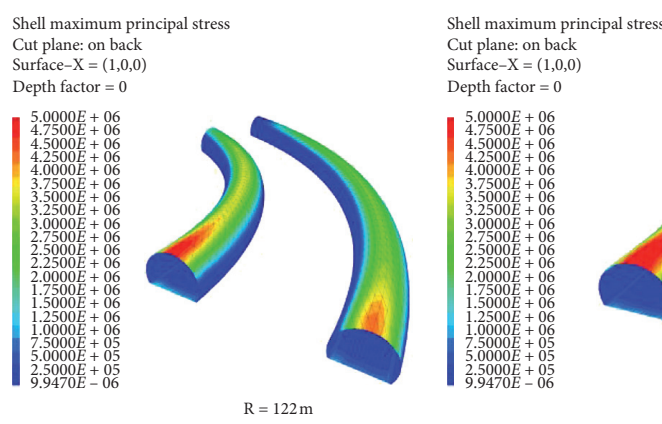

FIgURE 6: Maximum principal stress of tunnels with different curvature radii $(\mathrm{Pa})$.

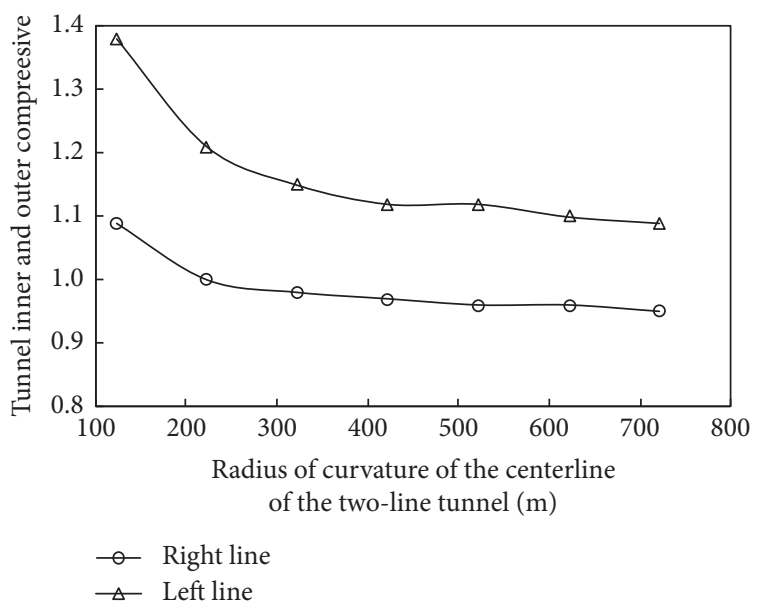

(a)

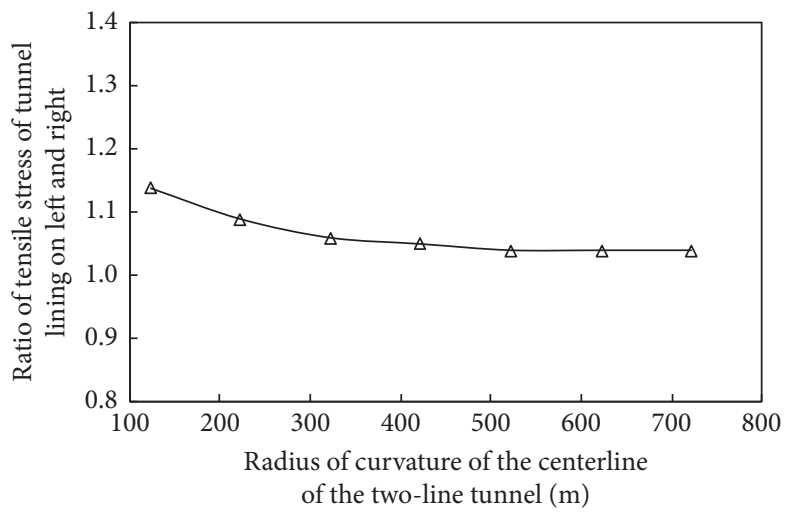

(b)

FIGURE 7: Variation curve of asymmetric stress ratio with the curvature radius.

\subsection{Influence on the Unevenness of Tunnel Deformation.} The small curvature radius of the tunnel causes the uneven deformation of the cross section of the double-line spiral tunnel. As shown in Figure 8, as the tunnel excavation depth increases, the amount of convergence deformation of the initial cross section gradually increases and tends to be stable. Among them, the distance between the two points $B$ and $C$ increases, and the distance between the two points $A$ and $\mathrm{B}$ and the distance between the two points $A$ and $C$ decrease, indicating that the entire section is deformed by transverse compression, and the vault $A$ settles downward. The vault settlement of the double-line spiral tunnel with a curvature radius of $122 \mathrm{~m}$ is greater than that with a radius of $722 \mathrm{~m}$.

As shown in Figures 8(a) and 8(b), when $R$ is $122 \mathrm{~m}$, the $\mathrm{AB}$ convergence deformation is less than $\mathrm{AC}$, indicating that the convergence deformation on both sides of the tunnel center line is uneven. The entire cross section deformation is compressed inward, and asymmetry is shown. It can be seen from the figure that the asymmetry of the inner line (left line) tunnel is greater than that of the outer line tunnel, which is consistent with the distribution law of compressive stress inside and outside the tunnel. As shown in Figure 8(c), when $R$ is $722 \mathrm{~m}$, the $\mathrm{AB}$ convergence deformation is slightly smaller than AC but very close, indicating that the convergence deformation unevenness on both sides of the tunnel center line is not obvious.

3.4. Influence on Deformation of Surrounding Rock. Figure 9 shows the vertical deformation cloud diagram of the tunnel surrounding rock when the curvature radius $R$ of the center line of the double spiral tunnel is $122 \mathrm{~m}$ and $722 \mathrm{~m}$, respectively. It can be seen from the figure that the clear distance between the left and right tunnels is greater than 2 times the diameter of the tunnel, so the mutual disturbance caused by the construction of the double-line tunnel is weak. At this time, two settlement grooves appear in the vertical deformation curve of the surrounding rock.

It should be noted that when $R$ is $122 \mathrm{~m}$, the deformation of the surrounding rock of the left and right tunnels shows an obvious asymmetry. Within the tunnel height range, the amount and range of vertical deformation of the surrounding rock of the inner tunnel are larger than those of the outer tunnel. As for the area above the tunnel vault, the vertical deformation range of the surrounding rock inside the inner tunnel is smaller than that outside the outer tunnel. Differently, when $R$ is $722 \mathrm{~m}$, the vertical deformation of the surrounding rock of the tunnel is a typical $W$-shaped symmetrical trend, which is in line with the trend of the 


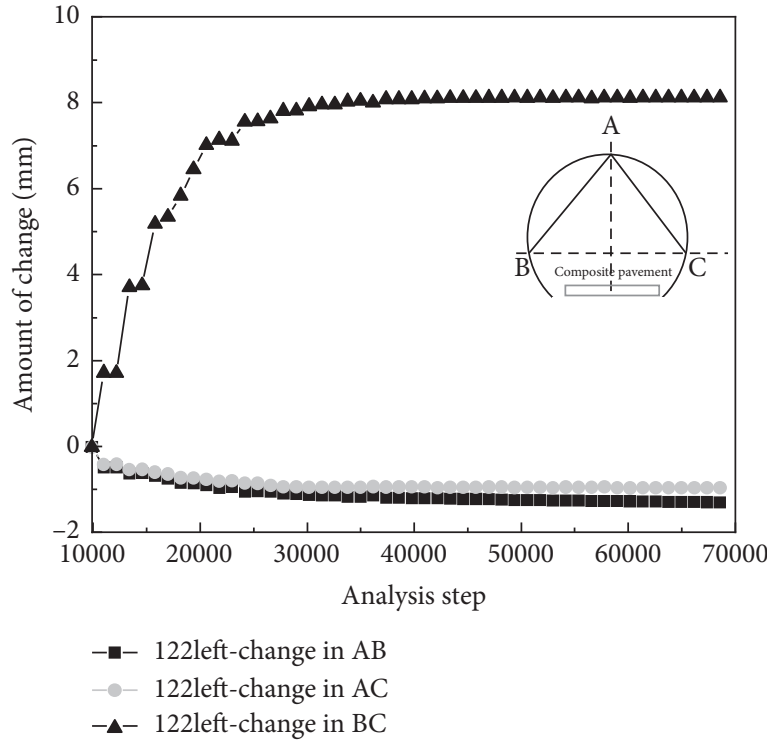

(a)

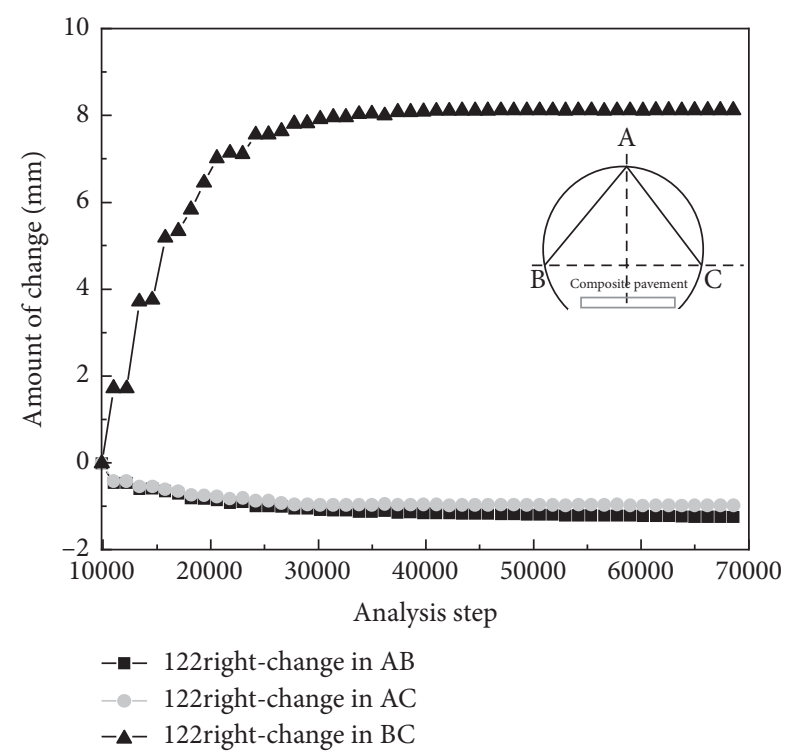

(b)

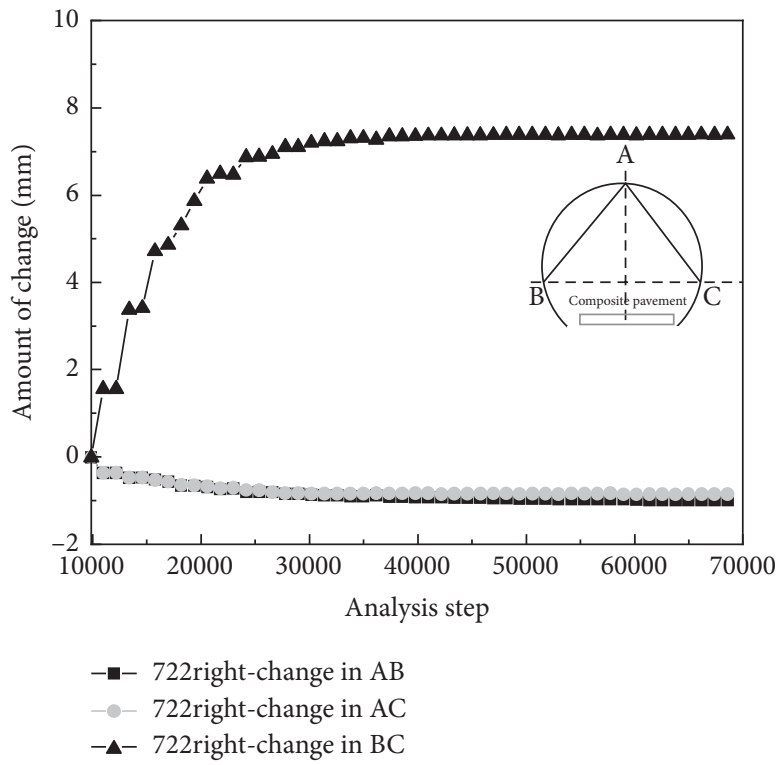

(c)

FIGURE 8: Convergent deformation curve of the tunnel cross section during construction.

Zone displacement magnitude

$$
\begin{aligned}
& 1.4375 E-02 \\
& 1.4000 E-02 \\
& 1.3000 E-02 \\
& 1.2000 E-02 \\
& 1.1000 E-02 \\
& 1.0000 E-02 \\
& 9.0000 E-03 \\
& 8.0000 E-03 \\
& 7.0000 E-03 \\
& 6.0000 E-03 \\
& 5.0000 E-03 \\
& 4.0000 E-03 \\
& 3.0000 E-03 \\
& 2.0000 E-03 \\
& 1.0000 E-03 \\
& 0.0000 E+00
\end{aligned}
$$

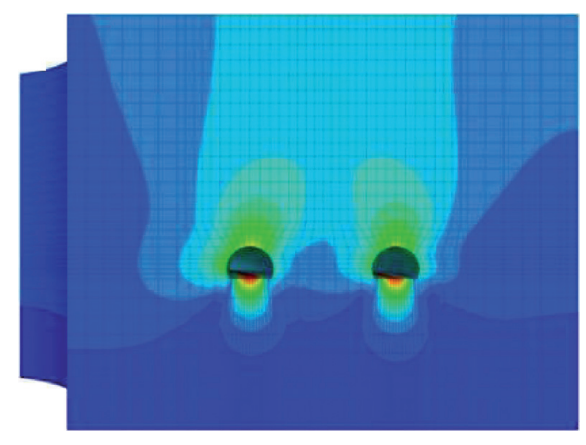

$\mathrm{R}=122 \mathrm{~m}$
Zone displacement magnitude

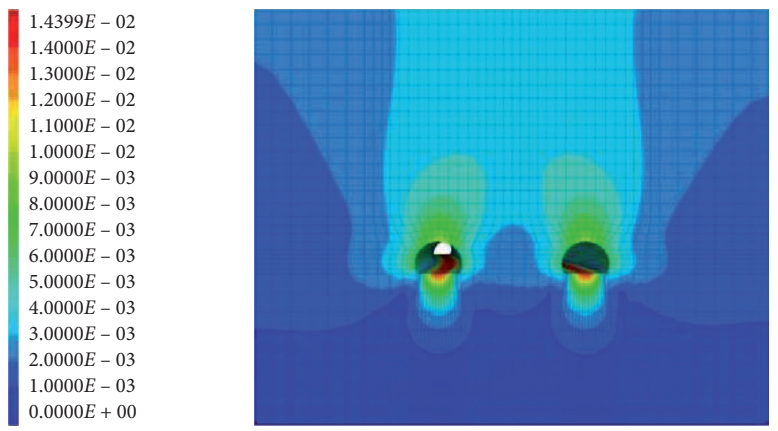

$\mathrm{R}=722 \mathrm{~m}$

FIgURE 9: Deformation of the tunnel surrounding rock (m). 
deformation of the surrounding rock for the separated double-line tunnel at straight-line alignment.

\section{Conclusions}

Based on the project of a double-line spiral tunnel in China's Yunnan province, a three-dimensional elastoplastic finite difference model of rock-tunnel was established in FLAC3D. Furthermore, by numerical simulation, the influence of the curvature radius on the mechanical behaviors of the doubleline spiral tunnel during the construction process is studied. The following conclusions can be obtained:

(1) When the curvature radius of the double-line spiral tunnel is small (about less than $200 \mathrm{~m}$ ), the stress distribution of tunnels shows asymmetry. The compressive stress at the internal side is greater than the external one. Compared with outside tunnel, the asymmetry of inside tunnel is more significant. As the curvature radius increases (approximately greater than $400 \mathrm{~m}$ ), the compressive stress ratio of the inside side to the outside side tends to be fixed. The smaller curvature radius results in the fact that the tensile stress at the vault of the inner tunnel is significantly larger than that of the outer tunnel.

(2) The convergence deformation of the tunnel cross section gradually increases and then tends to be stable as the construction process. Transverse compression deformation occurred on both cross sections of the double-line tunnel, and point $A$ at the vault settled downward. It should be noted that when the curvature radius of the spiral tunnel is small, asymmetry occurs in the convergence deformation on both sides of the tunnel center line. The entire cross section deformation is compressed inwards, and the asymmetry of the inner tunnel is greater than that of the outer tunnel. What is more, the small curvature radius results in a large settlement at vault of the tunnel.

(3) The distance between the left and right tunnels is greater than 2 times the diameter of the tunnel, so the mutual disturbance of the construction of the double-line tunnel is weak. Two settlement slots appear in the vertical deformation curve of the surrounding rock. When the curvature radius of the double-line spiral tunnel is small (about less than $200 \mathrm{~m}$ ), the deformation of the surrounding rock of the left and right tunnels is obviously asymmetric. The vertical deformation of the surrounding rock on top of the inner line tunnel is greater than that of the outer line tunnel. When the curvature radius is large, the vertical deformation curve of the surrounding rock is typical $\mathrm{W}$-shaped symmetrical, which is consistent with the trend of the deformation of the surrounding rock of the separated double-line tunnel at straight-line alignment.

The influence of the curvature radius of the tunnel alignment on the deformation and stress of the double-line spiral tunnel and its surrounding rock provides a reference for the construction stability control measures of the similar double-line spiral tunnel.

\section{Data Availability}

This is an open-access article distributed under the Creative Commons Attribution License, which permits unrestricted use, distribution, and reproduction in any medium, provided the original work is properly cited.

\section{Conflicts of Interest}

The authors declare that they have no conflicts of interest.

\section{References}

[1] Wogli G. Railway Network Switzerland. Swiss Federal Railways, General Secretariat, 1980.

[2] M. David, "Spiral tunnel runs rings around arctic circle dam site," Tunnels and Tunnelling International, vol. 16, no. 11, pp. 15-17, 1987.

[3] B. Chen, H. Ren, and L. X. Fu, "On the portal construction technology of spiral tunnel in special stratum," Applied Mechanics and Materials, vol. 94-96, pp. 1879-1884, 2011.

[4] N. Losacco and G. M. B. Viggiani, "Class A prediction of mechanised tunnelling in Rome," Tunnelling and Underground Space Technology, vol. 87, pp. 160-173, 2019.

[5] A. Paithankar, "Critical appraisal of double spiral cut in tunnel blasting," in Proceedings of International Society for Rock Mechanics and Rock Engineering International Symposium, pp. 370-388, Madrid, Spain, September 1988.

[6] F. Hao, "Analysis and prediction of uneven settlement due to a curved shield metro tunnel," Civil Engineering Research Journal, vol. 7, no. 4, Article ID 555720, 2019.

[7] M. Zhang, S. Li, and P. Li, "Numerical analysis of ground displacement and segmental stress and influence of yaw excavation loadings for a curved shield tunnel," Computers and Geotechnics, vol. 118, Article ID 103325, 2020.

[8] T. Huynh, M. Sugimoto, and J. Chen, "Study on steering method of $\mathrm{H} \& \mathrm{~V}$ shield to construct a spiral tunnel," in Proceedings of ITA WTC 2015 Congress, Dubrovnik, Croatia, May 2015.

[9] A. Alsahly, J. Stascheit, and G. Meschke, "Advanced finite element modeling of excavation and advancement processes in mechanized tunneling finite element modeling of excavation and advancement proesses in mechanized tunneling," Advances in Engineering Software, vol. 100, pp. 198-214, 2016.

[10] S. Miliziano and A. de Lillis, "Predicted and observed settlements induced by the mechanized tunnel excavation of metro line C near S. Giovanni station in Rome," Tunnelling and Underground Space Technology, vol. 86, pp. 236-246, 2019.

[11] T. N. Huynh, J. Chen, and M. Sugimoto, "Analysis on shield operational parameters to steer articulated shield," Japanese Geotechnical Society Special Publication, vol. 2, no. 42, pp. 1497-1500, 2016.

[12] T. Huynh, H. Pham, and M. Sugimoto, "Simulation of H\&V shield behavior at sharp curve by kinematic shield model," Geotechnical Engineering Journal of the SEAGS \& AGSSEA, vol. 2, no. 48, pp. 96-103, 2017.

[13] S. Mitsutaka, S. Aphichat, K. Shinji, and S. Yutaka, "Simulation of shield tunneling behavior along a curved alignment 
in a multilayered ground," Journal of Geotechnical and Geoenvironmental Engineering, vol. 133, no. 6, pp. 684-695, 2007.

[14] S. Li, G. Gu, P. Li, and M. Zhang, "Numerical simulation of mechanical properties of the segments for a curved shield tunnel during construction stage: a case study," in Proceedings of International Conference on Geotechnical and Earthquake Engineering, pp. 61-69, Chongqing, China, October 2018.

[15] X. Wu, "Safe construction technology of ganhaizi spiral tunnel," Southwest Highway, vol. 4, pp. 85-89, 2009, in Chinese.

[16] S. He, Y. Wu, and J. Zang, "Study on ventilation characteristics of high-drop spiral tunnel during operation period," in Proceedings of the 11th International Symposium on Heating, Ventilation and Air Conditioning (ISHVAC 2019), Singapore, March 2019.

[17] M.-B. Wang and S.-C. Li, "A complex variable solution for stress and displacement field around a lined circular tunnel at great depthfield around a lined circular tunnel at great depth," International Journal for Numerical and Analytical Methods in Geomechanics, vol. 33, no. 7, pp. 939-951, 2009.

[18] A.-z. Lu, L.-q. Zhang, and N. Zhang, "Analytic stress solutions for a circular pressure tunnel at pressure and great depth including support delay," International Journal of Rock Mechanics and Mining Sciences, vol. 48, no. 3, pp. 514-519, 2011.

[19] A. R. Kargar, R. Rahmannejad, and M. A. Hajabasi, "A semianalytical elastic solution for stress field of lined non-circular tunnels at great depth using complex variable methodfield of lined non-circular tunnels at great depth using complex variable method," International Journal of Solids and Structures, vol. 51, no. 6, pp. 1475-1482, 2014.

[20] A.-Z. Lu, N. Zhang, and L. Kuang, "Analytic solutions of stress and displacement for a non-circular tunnel at great depth including support delay," International Journal of Rock Mechanics and Mining Sciences, vol. 70, pp. 69-81, 2014.

[21] J. Fu, J. Yang, L. Yan, and S. M. Abbas, "An analytical solution for deforming twin-parallel tunnels in an elastic half plane," International Journal for Numerical and Analytical Methods in Geomechanics, vol. 39, no. 5, pp. 524-538, 2015.

[22] F. Kong, D. Lu, X. Du, and C. Shen, "Elastic analytical solution of shallow tunnel owing to twin tunnelling based on a unified displacement function," Applied Mathematical Modelling, vol. 68, pp. 422-442, 2019.

[23] J. P. Sahoo and J. Kumar, "Stability of long unsupported twin circular tunnels in soils," Tunnelling and Underground Space Technology, vol. 38, pp. 326-335, 2013.

[24] F. Yang and J. S. Yang, "Stability of shallow tunnel using rigid blocks and finite-element upper bound solutions," International Journal of Geomechanics, vol. 10, no. 6, pp. 242-247, 2010.

[25] Y. Zhang, X. Zhuang, and R. Lackner, "Stability analysis of shotcrete supported crown of NATM tunnels with discontinuity layout optimization," International Journal for $\mathrm{Nu}$ merical and Analytical Methods in Geomechanics, vol. 42, pp. 1-18, 2018.

[26] Z. Sun, Y. Zhang, Y. Yuan, and H. Mang, "Stability analysis of a fire-loaded shallow tunnel by means of a thermo-hydrochemo-mechanical model and discontinuity layout optimization," International Journal for Numerical and Analytical Methods in Geomechanics, vol. 43, pp. 1-14, 2019. 